\title{
Hydration dependent mechanical performance of denture adhesive hydrogels
}

\begin{abstract}
:
Objective. Hydration in denture adhesives regulates the formation of complex morphologies and mechanical function. Multiscale experimental approaches are required to evaluate the impact of hydration on the inherent heterogeneity of denture adhesive-based hydrogels at different length scales and the impact of such phenomena on adhesion performance.
\end{abstract}

Methods. The morphology of hydrated denture adhesives was examined via cryo-scanning electron microscopy (cryo-SEM). The rheological and thermodynamic behaviour of bulk hydrated deture adhesives was examined by rheology and differential scanning Calorimetry (DSC). The microscopic mechanical properties of the denture adhesives were characterised by atomic force microscopy (AFM) and compared to the properties measured at the macroscopic scale.

Results. The rheological and mechanical properties of commerically available denture adhesive hydrogels were found to be critically dependent on both the formulation of the adhesives and their hydration level. Clear progression of phase separation was observed in hydrated denture adhesives as hydration increased and changed the mechanical properties of the adhesives at multiple length scales. The adhesives displaying more heterogeneous structures, which were associated with the presence of 
hydrophobic and organic compounds in the formulation, exhibited more variable mechanical behaviour and weaker rheological properties, but stronger adhesive properties.

Significance. Our results are important in defining the relationships between hydrophilicity, hydration, mechanical and adhesive properties of denture adhesives, allowing the development of improved chemical formulations that control the fixation of dentures.

\section{Introduction}

Denture adhesives are commonly used to promote denture retention, stability and function in oral applications. These materials are usually based on macromolecules that can sustain the formation of numerous hydrogen bonds and contribute to the strong non-covalent bonding once mixed with mucus and saliva, to acquire viscous and adhesive properties [1]. Most of these adhesives contain both hydrophobic and hydrophilic molecules, which are designed to balance their bonding strengths to the gum and the denture [2-5]. Understanding the mechanical behaviour of the hydrated adhesives is required for the design of improved bioadhesives. Specifically, relationships between adhesive mechanical performance, formulation and hydration of denture adhesive are needed, in order to improve our control of the fixation of dentures. Our previous study highlighted marked differences in failure mechanisms of commercial denture adhesives (PDFP and PDAC)[6]. The existence of hydrophobic, 
organic compounds in the formulation of PDAC and the associated inherent heterogeneity of the resulting materials was proposed to be a critical factor responsible for the variation of bulk adhesive performance.

Hydration within the oral cavity is proposed to contribute to generate a complex morphology affecting the mechanical function of denture adhesives. Bioadhesives can absorb considerable amounts of water due to their hydrophilicity and therefore function is sensitive to hydration. From fundamental considerations, the hydration of the bioadhesives may support the formation of interlocked physical chain entanglement, electrostatic interactions and secondary chemical interactions (hydrogen bonding) that contribute to the bulk mechanical and rheological properties of the corresponding biomaterials [7-10]. Previous research also inferred that the hydrophilicity and solubility of some of the macromolecules within the adhesive composition could lead to leaching of molecules and reduce structural and functional properties of the adhesives [11]. Overly hydrophilic materials were found to lead to the fast deterioration of the mechanical properties of adhesives and to cause hydrolytic degradation [12-14]. However, a study of the role of hydration on the microstructure and mechanical properties of different types of denture adhesives is lacking. Therefore, a detailed understanding of the relationships between hydrophilicity, water uptake and mechanical and adhesive properties of the formulations currently used as denture adhesives is required [2, 3, 15], especially across a range of length scales shown to be critical in defining adhesive 
performance[6].

This work therefore aims to evaluate the impact of hydration on the inherent heterogeneity of denture adhesive-based hydrogels at different length scales and the impact of such phenomena on adhesion performance. The mechanical properties of hydrogel formulations used as denture adhesives, after hydration at different levels, were characterised via nanoscale indentation, by atomic force microscopy (AFM) [16-18]. The morphology of these hydrogels was visualised using scanning electron microscopy operating under sample cryogenic conditions (cryo-SEM). The influence of absorbed water and the level of hydration of the corresponding macromolecules was further quantified through differential scanning calorimetry (DSC).

\section{Materials and methods}

\subsection{Materials}

Two commercial adhesives, Poligrip ${ }^{\circledR}$, Ultra Wernets ${ }^{\circledR}$, Denture Fixative Powder (PDFP) and Poligrip ${ }^{\circledR}$, Ooze-Control Tip ${ }^{\circledR}$ Denture Adhesive Cream (PDAC) were studied in this work. These adhesives were selected because of their comparable compositions, but difference in types of formulation (PDFP is a powder whereas the PDAC is a cream). We made a mention of this in the introduction. PDFP powder is composed of poly(methylvinylether/maleic acid) sodium-calcium mixed partial salt, 
cellulose gum and aroma. PDAC cream consists of poly (methylvinylether/maleic acid) sodium-calcium mixed partial salt, petrolatum, cellulose gum, mineral oil, silica, poly (methylvinylether/mal2eic acid), flavour, Red 30 aluminium lake and Red 7 calcium lake. PDAC contains more hydrophobic compounds such as hydrocarbon vehicles (mineral oil and petrolatum) compared to PDFP, in addition to MVE/MA copolymer, which may affect the hydration of the polymers and gel formation resulting in different adhesion behaviour. In order to evaluate the influence of water added in the denture adhesive hydrogels, series of samples with different DI water weight ratios as illustrated in Table 1 were prepared. All the samples were examined immediately after the hydrogel was macroscopically homogeneous. For PDAC, due to its cream formulations, pure adhesive with no water mixed was examined first. Water was gradually added to the adhesives at a weight ratio of water to adhesives starting at 10:1, followed by higher water ratios of $1: 1,1: 2,1: 4$ and 1:10. For PDFP, only four water ratios $(1: 1,1: 2,1: 4$ and 1:10) were prepared since pure PDFP is dry powder.

Table 1. Weight ratios applied to the denture adhesives (PDAC and PDFP) mixed with DI water.

\begin{tabular}{ccccccc}
\hline Adhesives & \multicolumn{5}{c}{ Weight ratio of DI water } \\
\hline PDAC & No water & $10: 1$ & $1: 1$ & $1: 2$ & $1: 4$ & $1: 10$ \\
PDFP & $\mathrm{n} / \mathrm{a}$ & $\mathrm{n} / \mathrm{a}$ & $1: 1$ & $1: 2$ & $1: 4$ & $1: 10$ \\
\hline
\end{tabular}




\subsection{Cryo-SEM}

Cryo-SEM is a low temperature electron microscopy technique, which involves the examination of materials below ambient temperature (typically between -100 to $-170{ }^{\circ} \mathrm{C}$ ) and allows the structure and morphology of the sample to be better preserved and imaged in a hydrated and chemically unaltered state. Cooling of the system is achieved with liquid nitrogen. The denture adhesives were first fixed on the cryo-stage using the optimal cutting temperature (OCT) compound glue. The cryo-stage with adhesive samples was then brought to dip into liquid nitrogen to satisfy cryo-temperature $\left(-130^{\circ} \mathrm{C}\right)$ and transferred in vacuum to the cold-stage of the cryo-prechamber of the SEM system (Quanta 3D FEG, EU/USA). The adhesive sample was then cut horizontally to create a cross-sectional area facing the electron detector using a sharp blade equipped within the prechamber. Afterwards, the temperature in the prechamber was brought to $-90{ }^{\circ} \mathrm{C}$ to sublime ice off the surface of the sample. Argon was pumped into the prechamber and a thin layer of platinum/gold was deposited onto the sample surface through plasma sputtering. The sample was subsequently inserted into the main cryo-chamber of SEM, where it remained frozen during imaging.

\subsection{Rheological testing}

Oscillatory rheometry was used to characterise the macroscopic mechanical properties of denture adhesive hydrogels across a range of weight ratios of DI water. 
Measurements were carried out using a Discovery Hybrid Rheometer (DHR-3, TA) incorporating a $20 \mathrm{~mm}$ parallel plate in a Peltier plate steel geometry. The temperature of the system was maintained at $35^{\circ} \mathrm{C}$ throughout the experiment to simulate the conditions of a human oral cavity. Three stage procedures were performed to examine rheological behaviour of the hydrogels. The first procedure employed an oscillation amplitude test to determine the Linear Viscoelastic Region (LVR) of the sample by applying small stresses that result in minor displacements of the sample. This amplitude sweep involved applying the oscillatory stresses in a logarithmic ramp from 0.1 $\mathrm{Pa}$ to $100 \mathrm{~Pa}$ with 10 measurement points per decade, at a fixed frequency of $1 \mathrm{~Hz}$. The second procedure was an oscillation frequency test, which is capable of providing an insight into molecular motion mechanisms. To perform the frequency ramp, a small displacement of $10^{-4} \mathrm{rad}$ was applied to the sample using a logarithmic frequency sweep from $0.1 \mathrm{~Hz}$ to $100 \mathrm{~Hz}$ with 10 measurement points per decade. Finally, a stress relaxation procedure was carried out to assess how hydrated viscoelastic denture adhesives relieve stresses under constant strain over a period of time. The pre-set strain was $1 \%$.

\subsection{Nanoscale AFM indentation tests}

The nanoscale mechanical properties of denture adhesive hydrogels were evaluated by mechanical indentation in air using AFM (NTegra, NT-MDT, Russia). AFM allows both high resolution imaging of nano structures prior to mechanical testing $[16,19]$ 
and accurate resolution of forces required to deform a range of different materials [20, 21]. Two types of AFM tips with different spatial resolutions were used here for AFM measurements (Fig. 1): pyramidal AFM tip have dimensions on the nanometer length scale, whereas the modified colloidal AFM tips have dimensions in the micron length scale and were coated with a PMMA layer. One setup used a regular silicon nitride probe (ThermoMicroscopes, CA) with a tip radius of $50 \mathrm{~nm}$ and nominal spring constant of $0.1 \mathrm{~N} . \mathrm{m}^{-1}$, as illustrated in Fig. 2a and b. A second AFM tip configuration used a similar probe but with a PMMA-coated bead affixed to the apex of the AFM tip and a nominal spring constant of $0.38 \mathrm{~N} . \mathrm{m}^{-1}$. The schematic of this modified AFM tip setups are shown in Fig. 2c and d. The diameter of the PMMA-coated bead attached to the AFM tip was $3.53 \mu \mathrm{m}$ as determined by SEM observation shown in Fig. 2d. Such an attached bead on AFM tip configuration has been effective in measuring nanomechanical behaviour in dental adhesives previously [6] .

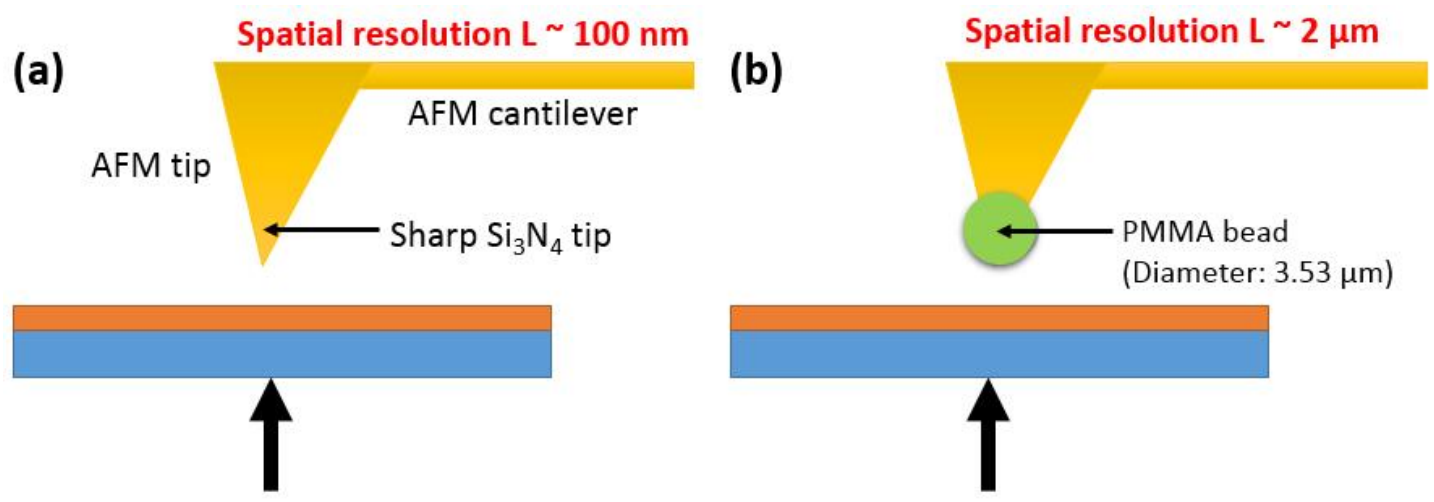

Figure 1. Schematic of the AFM mechanical indentation testing (a) using a regular AFM tip indenting the hydrogel sample and (b) using an AFM tip with PMMA-coated bead fixed to the tip indenting the sample. 

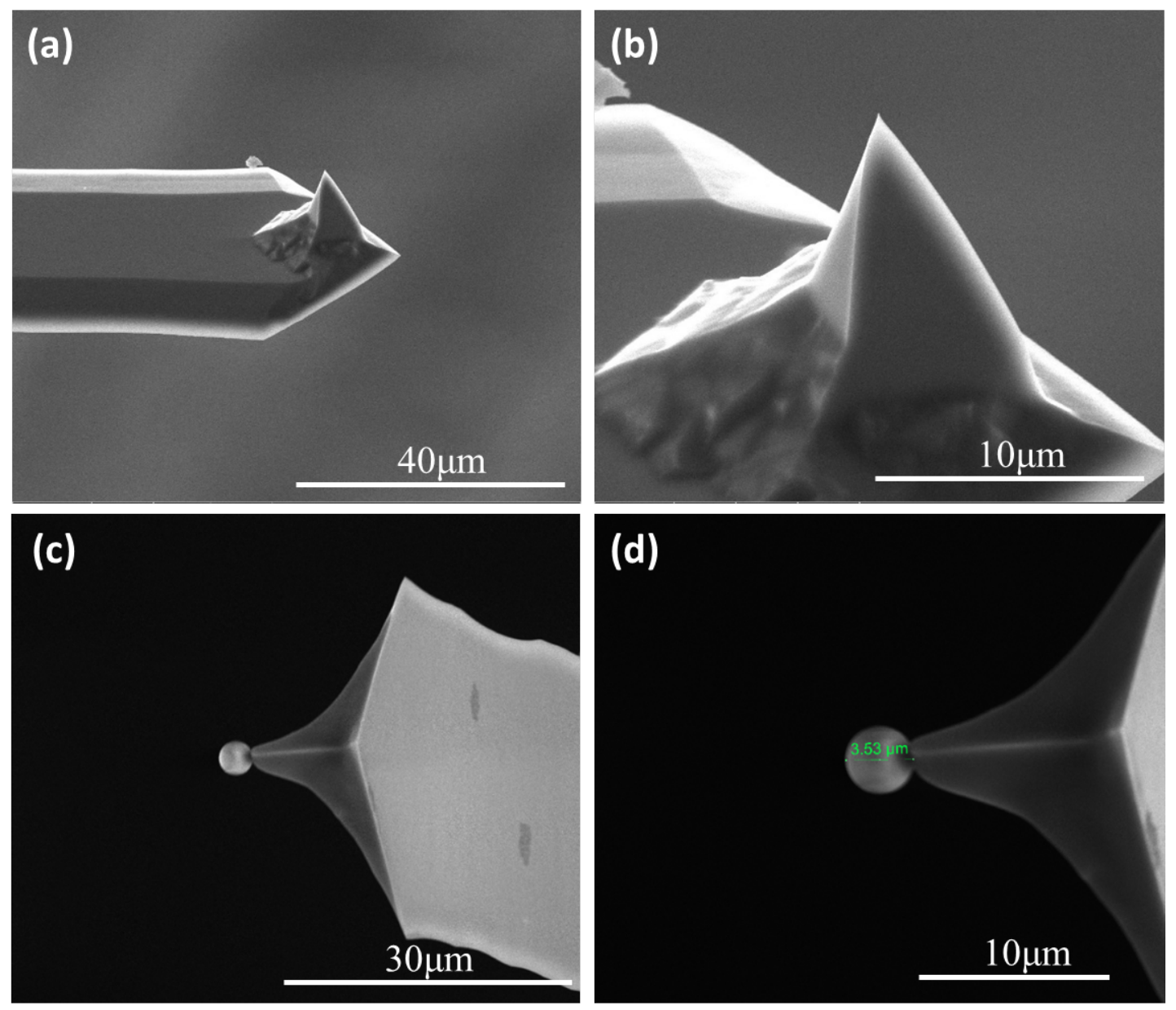

Figure 2. Scanning electron micrographs showing the AFM tip (a and b) and the AFM tip with the PMMA-coated bead at the apex of the tip (c and d). The diameter of the bead was approximately $3.53 \mu \mathrm{m}$.

The Young's moduli of denture adhesive hydrogels were determined by force spectroscopy at three different areas on adhesive hydrogels, probed by recording a force map across $50 \times 50 \mu \mathrm{m}^{2}$ area. Each force map consists of 100 force curves taken across a $10 \times 10$ array over this $50 \times 50 \mu \mathrm{m}^{2}$ area, giving 300 force curves in total for each adhesive gels. The Young's modulus of the adhesive gels were determined at two different length scales by indenting using the regular AFM tip (radius of 
curvature $\mathrm{R}=50 \mathrm{~nm}$ ) with an indentation depth of $\sim 100 \mathrm{~nm}$ and the bead modified tip (radius of curvature $\mathrm{R}=1.77 \mu \mathrm{m}$ ) with an indentation depth of $\sim 300 \mathrm{~nm}$. For the measurements with the regular tip, the spatial resolution (determined by the indentation depth and the geometry of the tip) was $\mathrm{L} \sim 100 \mathrm{~nm}$, whereas for bead modified tip used a spatial resolution was $\mathrm{L} \sim 2 \mu \mathrm{m}$. The Young's modulus and adhesion of the adhesive hydrogels were therefore estimated from the force-displacement curves obtained by analysing the recorded deflection signal of the cantilever during the tip approaching and retraction from each sample in the indentation process (see supporting information Fig. S1). Indentations of the AFM tip with the adhesive gels was carried out without creep steps, to provide information on the elasticity of the sample, without considering the effect of creep on the maximum load. The elasticity was calculated from the slope of the force-deflection curve measured against a reference surface to the nominal spring constant of the cantilever. A silicon wafer was used as the reference surface for measurements. The detailed calculation of the Young's moduli is provided as Supporting Information.

\subsection{Characterisation of water crystallisation}

Differential Scanning Calorimetry (DSC) was carried out on a callorimeter equipped with a cooling cell system that was used to quantify the crystallisation and melting of water contained in the adhesive hydrogels. The two denture adhesives examined here were prepared with the same weight ratios of DI water as in the SEM, rheological and 
AFM tests. The adhesive samples were weighted (typically 5-25 mg) and sealed in an aluminium pan with a small hole on the cap. The experiment was performed by cooling the sample from room temperature to $-50^{\circ} \mathrm{C}$ at the rate of $5^{\circ} \mathrm{C} \min ^{-1}$ and then heating the sample to room temperature at the same rate. The calibration of crystallization and melting temperature of the absorbed water was performed by repeating the cooling and heating procedures on pure DI water. The measurable water content in the sample was therefore calculated as water $\%=W_{\text {water }} / W_{\text {sample }}$, where $W_{\text {water }}$ is the weight of crystallisable water calculated from the enthalpy of melting peak in the DSC curve and $W_{\text {sample }}$ is the weight of the sample [22].

\section{Results}

\subsection{Cryo-SEM}

Fig. 3 Shows the scanning electron micrographs of the cross-sectional surfaces of the two denture hydrogels with varying water ratios at different magnifications, imaged at low temperature $\left(-130^{\circ} \mathrm{C}\right)$. The morphology of unhydrated PDAC shows a complex non-porous microstructure with no obvious phase separation. Fig.4 Illustrates the relationship between pore sizes and hydration in the two denture adhesives studied. Increases of the added weight ratio of DI water in the adhesive hydrogels provide a distinct observable phase separation, pore sizes increasing progressively. Noticeably, distinct porous structures appear in PDAC when the water content ratio is increased to 1:2, whereas no clear formation of different phases is observed in PDFP with the same 
water content.

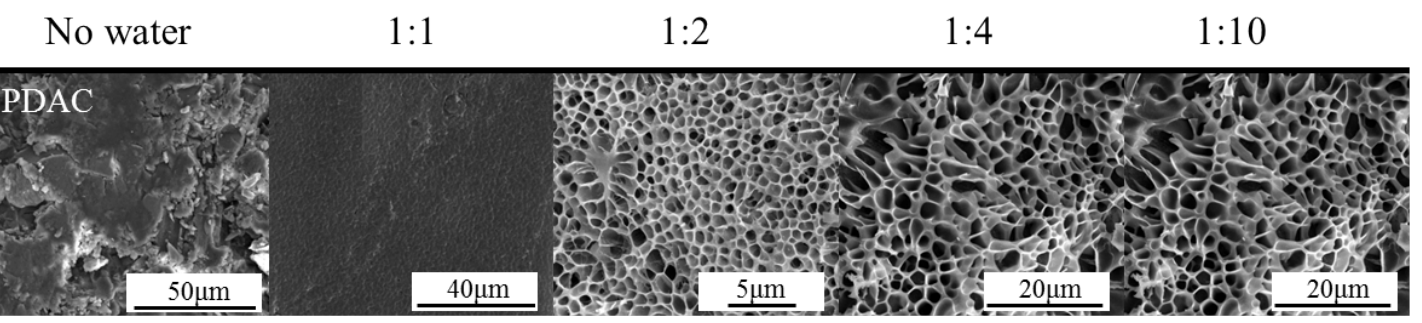

PDFP
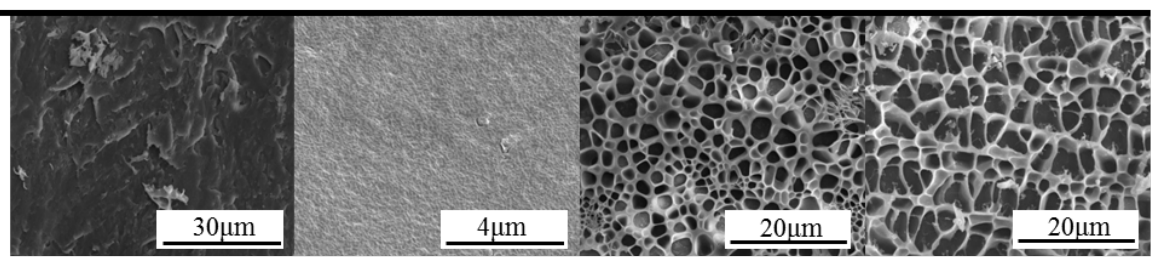

Figure 3. Scanning electron micrographs showing the cross-sectional morphologies of PDAC and PDFP hydrogels with different weight ratios of water. Note that pure PDFP is a powder and was not imaged dry by SEM.

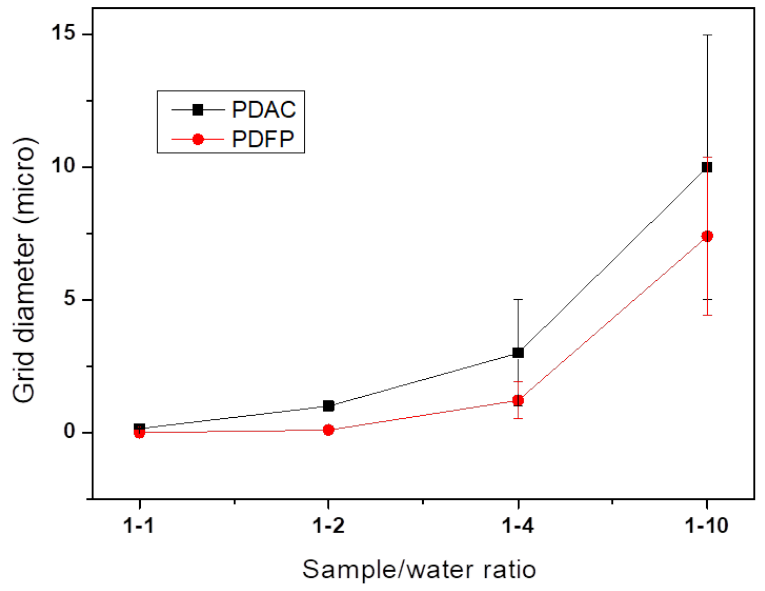

Figure 4. Relationship between pore size and hydration in the two adhesives studied.

\subsection{Rheological testing}

Rheological properties of the denture adhesive hydrogels were characterised from frequency sweeps and oscillation stress sweeps, as shown in Fig. 5. Oscillatory stress 
sweeps were performed to determine the linear viscoelastic region (LCR) for all denture adhesive hydrogels to ensure that the constant stress applied to all samples in frequency sweeps is within the LVR region. Fig. 5 (a) and (c) show the frequency sweeps of PDFP and PDAC, whereas Fig. 5 (b) and (d) show the results of oscillation amplitude sweeps. Pure PDAC was not examined by Rheometer because denture adhesives function in hydrated conditions. Generally, both denture adhesive hydrogels show clear transitions of storage shear modulus decreasing as more water is introduced into the mixture, indicating reasonably lower moduli for the denture adhesive mixture containing more water [12]. PDFP exhibits storage shear moduli approximately one order of magnitude higher than PDAC ranging from low water ratio to high water ratio according to both frequency and amplitude sweeps. Specifically, in Fig. 5 (a) and (c), adhesive hydrogels with high water ratios appear to fail quickly at high frequencies (up to $100 \mathrm{~Hz}$ ), due to the collapse of the discontinuous porous structure evidenced in cryo-SEM. This shear thinning behaviour is more pronounced for PDAC gels and marginal for PDFP, indicating PDAC appears stronger frequency dependent behaviour than PDFP. The increased shear thinning behaviour, leading to failure, is also observed at high oscillation stress in amplitude sweeps at high water ratios, as shown in Fig. 5 (b) and (d). Fig. 6 further displays the results of stress relaxation experiments. A clear decrease of initial stress is observed as water content increases for all measured adhesives. The maximum stress for PDFP generally exhibits one order of magnitude increase compared to PDAC, which is consistent with the results from frequency and amplitude sweeps as shown in Fig. 5. 
Frequency sweeps
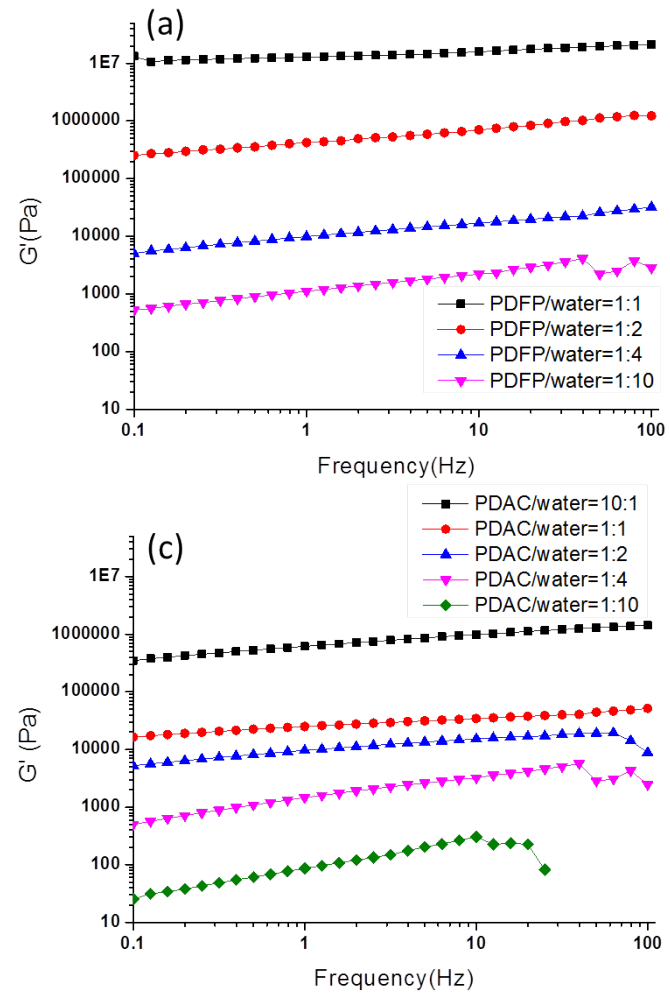

Osc. Stress sweeps
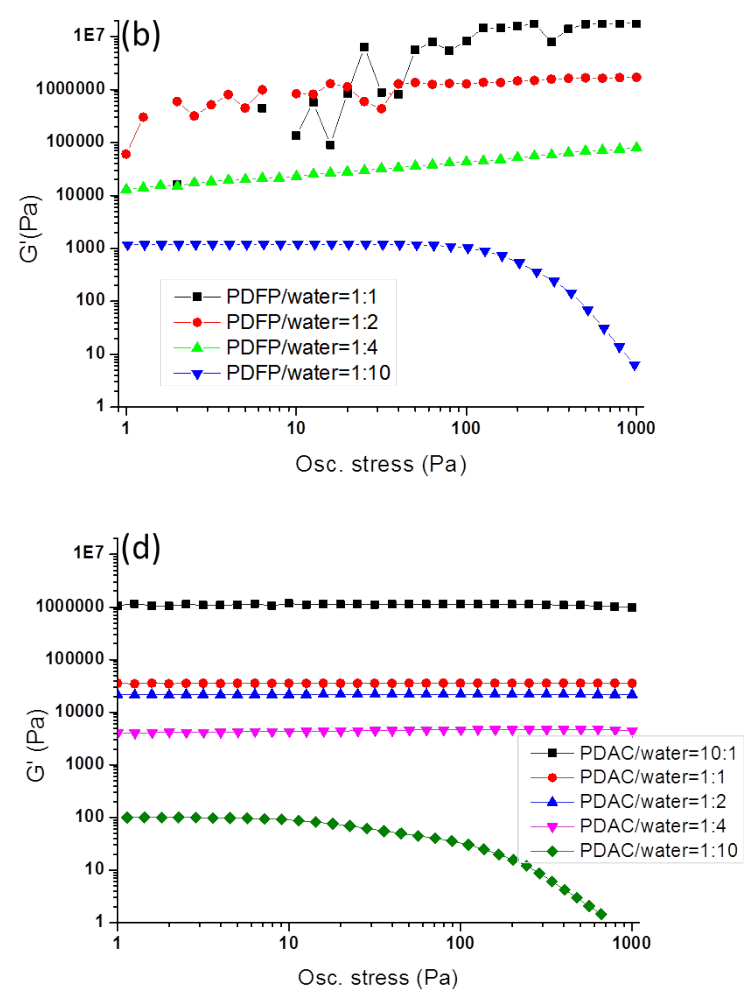

Figure 5. Plots of oscillation stress sweeps and frequency sweeps for PDAC and PDFP hydrogels.
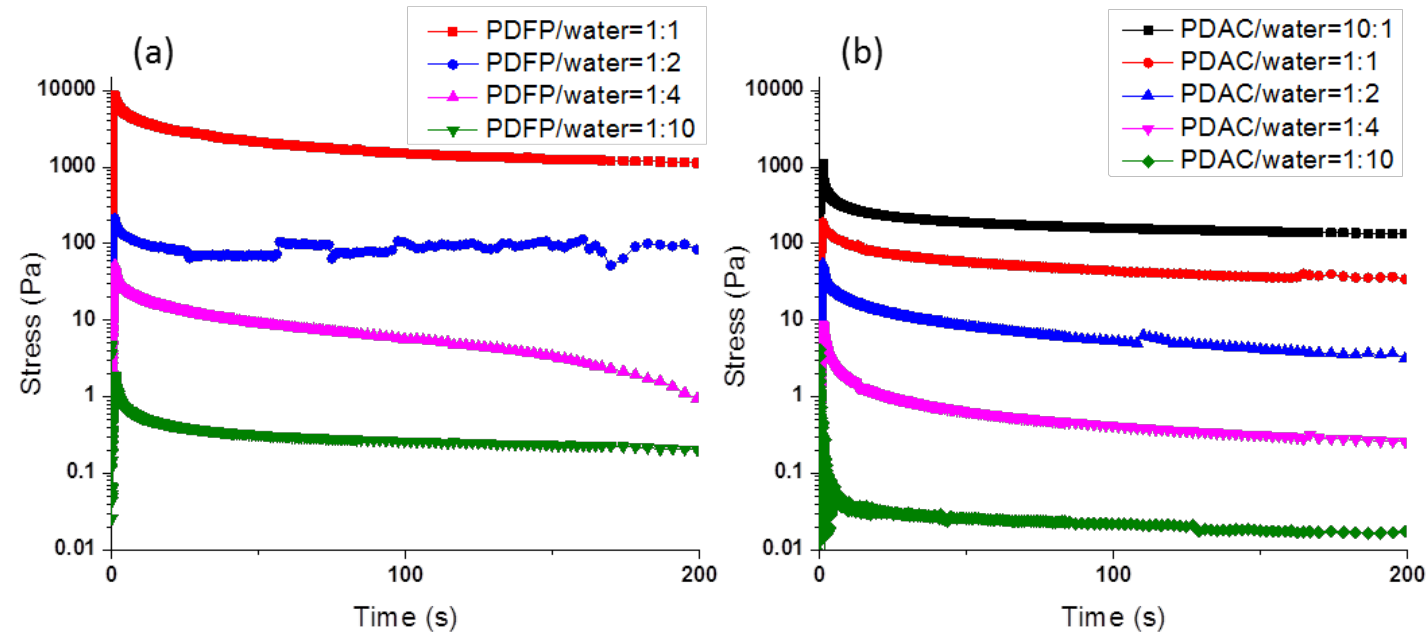

Figure 6. Plots of stress relaxation of PDFP and PDAC across a range of hydration conditions. 


\subsection{Nanoscale AFM indentation tests}

The nanoscale mechanical properties of the denture adhesive gels were investigated in order to gain further insight into their complex rheological and elastic properties. The Young's moduli of these denture adhesives, obtained from AFM nanoindentation with both conventional tips and PMMA-coated tips, are presented in Fig. 7. Distributions of Young's moduli of all hydrogel samples were also characterised by their relative standard deviation (RSD). Both denture adhesive hydrogels show decreased stiffness as water concentration increases, whereas similar Young's moduli were obtained from the two tips for all adhesive hydrogels. PDAC exhibited a Young's modulus approximately 1.5 times higher than that of PDFP, a difference that was associated with increased variability of the measurements and spread of the data. The distributions of the Young's moduli of denture adhesive gels measured by AFM on the spatial resolution of $\mathrm{L} \sim 2 \mu \mathrm{m}$ are less broad than those on the spatial resolution of $\mathrm{L} \sim 100 \mathrm{~nm}$ according to the RSD values summarised in Table 2.
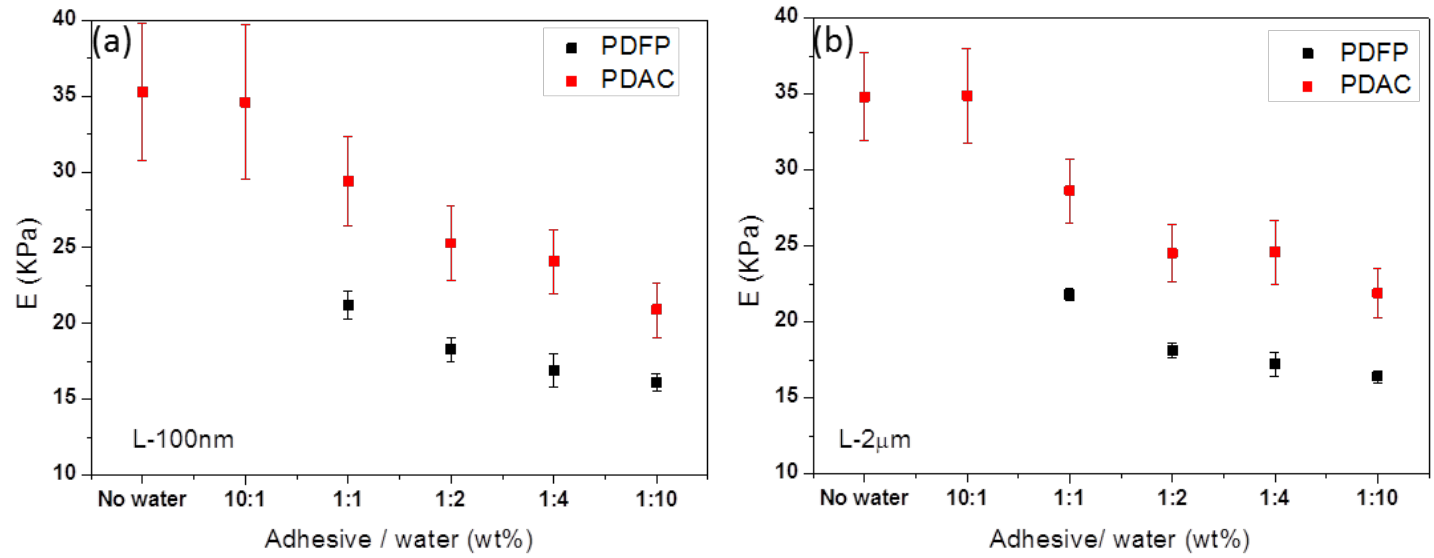

Figure 7. Young's moduli of the denture adhesive hydrogels at different water ratios. 
(a) $\mathrm{Si}_{3} \mathrm{~N}_{4}$ tip; (b) Tip attached with PMMA-coated bead.

Table 2. Summary of Young's moduli, E of PDFP and PDAC hydrogels at different weight ratios of water, measured by AFM at two different length scales $(\mathrm{L} \sim 100 \mathrm{~nm}$ and $\mathrm{L} \sim 2 \mu \mathrm{m})$.

\begin{tabular}{|c|c|c|c|}
\hline Sample & Ratio & $\mathrm{E}_{\mathrm{AFM}}(\mathrm{kPa}) / \mathrm{L} \sim 100 \mathrm{~nm}$ & $\mathrm{E}_{\mathrm{AFM}}(\mathrm{kPa}) / \mathrm{L} \sim 2 \mu \mathrm{m}$ \\
\hline \multirow[t]{4}{*}{ PDFP } & $1: 1$ & $21.2(0.90)(\mathrm{RSD}=4.2 \%)$ & $21.8(0.40)(\mathrm{RSD}=1.8 \%)$ \\
\hline & $1: 2$ & $18.3(0.80)(\mathrm{RSD}=4.4 \%)$ & $18.1(0.50)(\mathrm{RSD}=2.8 \%)$ \\
\hline & $1: 4$ & $16.9(1.10)(\mathrm{RSD}=6.5 \%)$ & $17.2(0.80)(\mathrm{RSD}=4.7 \%)$ \\
\hline & $1: 10$ & $16.1(0.60)(\mathrm{RSD}=3.7 \%)$ & $16.4(0.40)(\mathrm{RSD}=2.4 \%)$ \\
\hline \multirow[t]{7}{*}{ PDAC } & No water & $35.3(4.50)(\mathrm{RSD}=12.8 \%)$ & $34.8(2.90)(\mathrm{RSD}=8.3 \%)$ \\
\hline & $10: 1$ & $34.6(5.10)(\mathrm{RSD}=14.7 \%)$ & $34.9(3.10)(\mathrm{RSD}=8.9 \%)$ \\
\hline & $1: 1$ & $29.4(2.90)(\mathrm{RSD}=9.9 \%)$ & $28.6(2.10)(\mathrm{RSD}=7.3 \%)$ \\
\hline & $1: 2$ & $25.3(2.50)(\mathrm{RSD}=9.8 \%)$ & $24.5(1.90)(\mathrm{RSD}=7.8 \%)$ \\
\hline & $1: 4$ & $24.1(2.10)(\mathrm{RSD}=8.7 \%)$ & $24.6(2.10)(\mathrm{RSD}=8.5 \%)$ \\
\hline & $1: 10$ & $20.9(1.80)(\mathrm{RSD}=8.6 \%)$ & $(1.60)$ \\
\hline & & & $(\mathrm{RSD}=7.3 \%)$ \\
\hline
\end{tabular}

\subsection{Quantification of water crystallisability}

The thermodynamic behaviour of the hydrated adhesive mixtures was examined by DSC as shown in Fig. 8. The dashed line represents DSC heating curve of pure DI water. Compared with pure water, a broad melting peak appears between $-15^{\circ} \mathrm{C}$ to 
$10^{\circ} \mathrm{C}$ in adhesive hydrogels and the peak of melting shifts to a lower range of temperatures with decreasing contents of absorbed water. The content of the detectable freezable bound and free water [23] in denture adhesives increased progressively as the hydration level increased but was always lower than the total water content as shown in Fig. 8c.

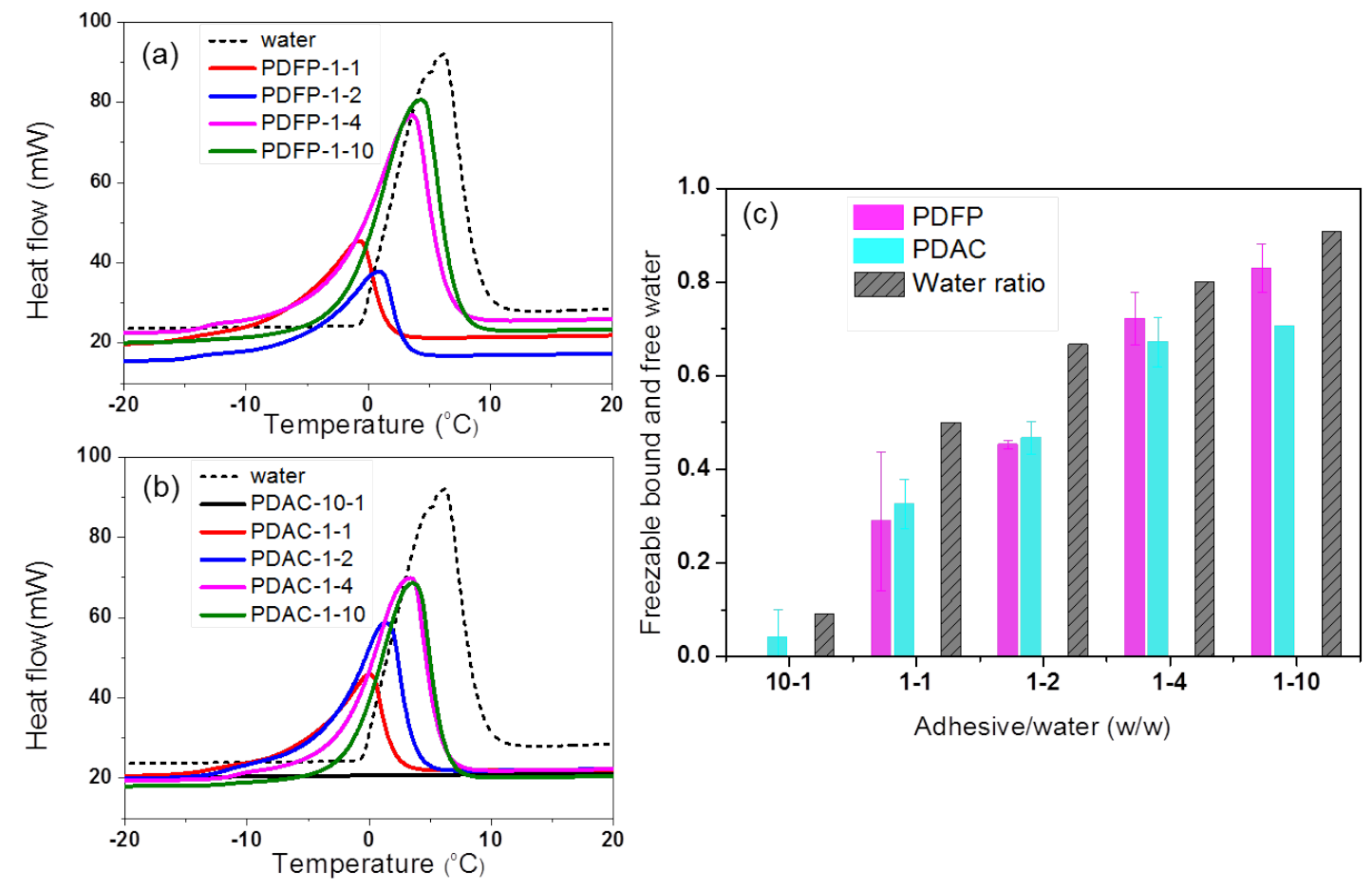

Figure 8. DSC heating curves of adhesive hydrogels with DI water at different weight ratios: (a) PDFP; (b) PDAC. Heating and cooling rate was $5^{\circ} \mathrm{C} / \mathrm{min}$. Dash line-pure water. (c) The relationship between the contents of freezable bound and free water contained in the hydrogels and adhesive/water ratios.

\section{Discussion}

The multiphasic morphologies of the hydrated denture adhesives shown in Fig. 3 
suggest that PDAC hydrogel exhibits more heterogeneities in the structure compared to PDFP. This could be presumable due to the fact that PDAC contains more hydrophobic hydrocarbons. The results of stronger shear moduli in PDFP than PDAC shown in Fig. 5 also prove this assumption. The cream formulation in PDAC soften the adhesive structure, whereas PDFP does not include such compounds in its structure and ,as a result, is not expected to be associated with microscale phase separation. This phenomenon is also associated with stronger frequency dependency of the PDAC adhesives shear moduli compared to PDFP (shown in Fig. 5a, c), especially at the highest hydration levels, which further indicates the formation of porous networks. Further, PDFP gels display modest relaxation level than PDAC in Fig. 6 and indicate a relatively high degree of crosslinking and entanglement. The hydrophobic viscous components in PDAC (mineral oil and petrolatum), otherwise, provide weak interfaces between these immiscible phases. As shown in Table 2, the increased variability in the local elastic moduli of PDAC compared to PDFP is thought to be indicative of its heterogeneous structure. Specifically, an AFM tip contacting a homogeneous gel structure is expected to give consistent mechanical behaviour, as exhibited in PDFP, whereas the heterogeneous structure of PDAC presents an inhomogeneous contact surface to the AFM tip during the indentation process. This can be further demonstrated by the result that the distributions of the Young's moduli of denture adhesive gels on spatial resolution of $\mathrm{L} \sim 2 \mu \mathrm{m}$ are less broad than those on the spatial resolution of $\mathrm{L} \sim 100 \mathrm{~nm}$ in Fig. 7. If considering the spatial resolution of these measurements, $\mathrm{L} \sim 2 \mu \mathrm{m}$, is larger than the scale of gel 
heterogeneities, the measured Young's moduli would average over many different local structural domains.

The addition of water significantly changes the morphologies and properties of the denture adhesives. The properties of the adhesive hydrogels decrease dramatically as water ratio increases according to both rheological and mechanical results, due to the associated dilution of cross-linking moieties and decreased macromolecular entanglement. The porous structures shown in Fig. 3 appear discontinuous at high water ratios. These hydrogels with high water ratios attempt to fail quickly at high frequencies (Fig. 5 a and c) potentially due to the collapse of the discontinuous porous structure. The results from DSC (Fig. 8a and b) suggest that, at low hydration levels, the absorbed water has a perturbed destabilised structure (lower melting point) and that, as the hydration level increases, increasing amounts of free water arise, not closely associated with macromolecules or interfaces [12]. The fact that detectable content of water is always lower than the total water added confirms the bonding of water molecules with macromolecules and other components of these materials (shown in Fig. 8c). Interestingly, PDFP shows a slightly lower content of detectable crystallisable water than PDAC when adhesive/water ratio was $1: 1$, but as this ratio increased to $1: 10$, the detectable crystallisable water in PDFP is clearly higher than that in PDAC, implying that water molecules form comparable stronger intermolecular bonds with the adhesive molecules in the macromolecular structure of PDAC hydrogels at high water ratios. 


\section{Conclusions}

The rheological and mechanical properties of the commercially available denture adhesive hydrogels are shown to be critically dependent on both the formulation of the adhesives and their hydration level. The evolution of structural heterogeneities in PDAC hydrogels provided more variable mechanical behaviour and lower rheological properties compared to the more homogenous PDFP hydrogel. Cryo-SEM images of hydrated denture adhesive hydrogels evidenced clear progression of the microstructure of these materials as hydration increases. These changes were found to be associated with striking changes in mechanical properties of the hydrogels, at multiple length scales. Compared with PDFP, PDAC hydrogels displayed more heterogenous structures and exhibited lower shear modulus and broader distributions of mechanical properties. The inherent heterogeneity of PDAC hydrogels is believed to be related to the presence of hydrophobic organic compounds, whereas PDFP hydrogels maintain a more homogeneous structure due to the good hydrophilicity of all of its components. Therefore, PDFP displays stiffer mechanical properties, whilst PDAC displays a stronger adhesive strength to denture surfaces, as presented in our previous study [6]. Our results indicate that the heterogeneous structure of PDAC creams, although softening the corresponding hydrated hydrogels and conferring a more pronounced viscoelastic response, allows hydrophobic domains to form stronger adhesion with hydrophobic denture surfaces. In addition, the viscoelastic response of adhesive hydrogels is proposed to contribute to their performance by absorbing some of the shock energy associated with mastication. Hence our study, complemented by 
patient surveys quantifying the performance of these materials, provides guidelines for the rational design of novel denture adhesives.

\section{Reference}

1. Madsen, F., K. Eberth, and J.D. Smart A rheological assessment of the nature of interactions between mucoadhesive polymers and a homogenised mucus gel. Biomaterials, 1998; 19: 1083-92.

2. Yiu, C.K., N.M. King, D.H. Pashley, B.I. Suh, R.M. Carvalho, M.R. Carrilho, and F.R. Tay Effect of resin hydrophilicity and water storage on resin strength. Biomaterials, 2004; 25: 5789-96.

3. Unemori, M., Y. Matsuya, S. Matsuya, A. Akashi, and A. Akamine Water absorption of poly(methyl methacrylate) containing 4-methacryloxyethyl trimellitic anhydride. Biomaterials, 2003; 24: 1381-1387.

4. Haller, B. Recent developments in dentin bonding. Am J Dent, 2000; 13: 44-50.

5. Nakabayashi, N., M. Takeyama, K. Kojima, and E. Masuhara Studies on dental self-curing resins (20)-Adhesion of 4-META/MMA-TBB resin to pretreated dentine. Shika Rikōgaku Zasshi 1982; 23: 29-33.

6. An, Y., D. Li, N. Roohpour, J.E. Gautrot, and A.H. Barber Failure mechanisms in denture adhesives. Dent Mater, 2016; 32: 615-623. 
7. Mortazavi, S.A., B.G. Carpenter, and J.D. Smart A comparative study on the role played by mucus glycoproteins in the rheological behaviour of the mucoadhesive/mucosal interface. Int J Pharm, 1993; 94: 195-201.

8. Hassan, E.E. and J.M. Gallo A simple rheological method for the in vitro assessment of mucin-polymer bioadhesive bond strength. Pharm Res, 1990; 7: 491-5.

9. Rossi, S., M.C. Bonferoni, G. Lippoli, M. Bertoni, F. Ferrari, C. Caramella, and U. Conte Influence of mucin type on polymer-mucin rheological interactions. Biomaterials, 1995; 16: 1073-9.

10. Mortazavi, S.A., B.G. Carpenter, and J.D. Smart An Investigation of the rheological behavior of the mucoadhesive mucosal interface. Int J Pharm, 1992; 83: 221-225.

11. Malacarne, J., R.M. Carvalho, M.F. de Goes, N. Svizero, D.H. Pashley, F.R. Tay, C.K. Yiu, and M.R. Carrilho Water sorption/solubility of dental adhesive resins. Dent Mater, 2006; 22: 973.

12. Ito, S., M. Hashimoto, B. Wadgaonkar, N. Svizero, R.M. Carvalho, C. Yiu, F.A. Rueggeberg, S. Foulger, T. Saito, Y. Nishitani, M. Yoshiyama, F.R. Tay, and D.H. Pashley Effects of resin hydrophilicity on water sorption and changes in modulus of elasticity. Biomaterials, 2005; 26: 6449-59.

13. Wolff, E.G. Moisture effects on polymer matrix composites. Sampe J, 1993; 29: $11-19$.

14. Musto, P., G. Ragosta, G. Scarinzi, and L. Mascia Probing the molecular interactions 
in the diffusion of water through epoxy and epoxy\&ndash;bismaleimide networks. J Polym Sci Pol Phys, 2002; 40: 922-938.

15. Burrow, M.F., S. Inokoshi, and J. Tagami Water sorption of several bonding resins. Am J Dent, 1999; 12: 295-8.

16. Wang, W., A.J. Bushby, and A.H. Barber Nanomechanical thermal analysis of electrospun polymer fibers. Appl Phys Lett, 2008; 93: 201907.

17. Kao, A.P., J.T. Connelly, and A.H. Barber 3D nanomechanical evaluations of dermal structures in skin. J. Mech Behav Biomed Mater, 2016; 57: 14-23.

18. Grant, C.A., P.C. Twigg, and D.J. Tobin Static and dynamic nanomechanical properties of human skin tissue using atomic force microscopy: effect of scarring in the upper dermis. Acta biomater, 2012; 8: 4123-4129.

19. Magonov, S.N. and M.H. Whangbo Surface analysis with STM and AFM. Angew Chem Int Edit, 1996; 109: 1415-1416.

20. Barber, A.H., R. Andrews, L.S. Schadler, and H. Daniel Wagner On the tensile strength distribution of multiwalled carbon nanotubes. Appl Phys Lett, 2005; 87: 1297.

21. Barber, A.H., S.R. Cohen, and H.D. Wagner Measurement of carbon nanotubepolymer interfacial strength. Appl Phys Lett, 2003; 82: 4140-4142.

22. Nakamura, K., T. Hatakeyama, and H. Hatakeyama Studies on bound water of cellulose by differential scanning calorimetry. Text Res J, 1981; 51: 607-613. 
23. Cotugno, S., D. Larobina, G. Mensitieri, P. Musto, and G. Ragosta A novel spectroscopic approach to investigate transport processes in polymers: the case of water-epoxy system. Polymer, 2001; 42: 6431-6438. 